Meta

Journal des traducteurs

Translators' Journal

\title{
Terminologie et documentation
}

\section{Guy Rondeau}

Volume 25, numéro 1, mars 1980

La documentation

URI : https://id.erudit.org/iderudit/003983ar

DOI : https://doi.org/10.7202/003983ar

Aller au sommaire du numéro

Éditeur(s)

Les Presses de l'Université de Montréal

ISSN

0026-0452 (imprimé)

1492-1421 (numérique)

Découvrir la revue

Citer cet article

Rondeau, G. (1980). Terminologie et documentation. Meta, 25(1), 152-170.

https://doi.org/10.7202/003983ar

Ce document est protégé par la loi sur le droit d'auteur. L'utilisation des services d'Érudit (y compris la reproduction) est assujettie à sa politique d'utilisation que vous pouvez consulter en ligne.

https://apropos.erudit.org/fr/usagers/politique-dutilisation/
Cet article est diffusé et préservé par Érudit.

Érudit est un consortium interuniversitaire sans but lucratif composé de l’Université de Montréal, l'Université Laval et l'Université du Québec à Montréal. Il a pour mission la promotion et la valorisation de la recherche. https://www.erudit.org/fr/ 


\title{
Terminologie et documentation*
}

\author{
GUY RONDEAU \\ professeur,
}

Université Laval

\section{Introduction}

0.1 Terminologie et documentation sont indissociables l'un de l'autre, en ce sens qu'il est impossible de faire de la terminologie sans avoir accès, directement ou indirectement, à une abondante documentation spécialisée.

En effet, l'objet linguistique propre de la terminologie est le terme; or, les termes se trouvent soit en vrac dans l'immense corpus que constituent les communications scientifiques ou techniques (CST) soit sous une forme quelconque de classement dans des ouvrages lexicographiques de tous ordres (glossaires, vocabulaires, dictionnaires, banques de termes, etc.).

Il en résulte que le terminologue doit non seulement avoir la possibilité de consulter une documentation complète, mais qu'il doit également être en mesure de juger de la valeur et de la fiabilité des documents dont il se sert et savoir exploiter la documentation. Cette dernière question relève cependant de la formation du terminologue; c'est pourquoi elle ne sera abordée ici que de façon sommaire.

0.2 Par ailleurs, si les CST peuvent se présenter sous forme sonore aussi bien que sous forme écrite, les méthodes de travail de la terminologie favorisent le support fourni par l'écriture ; ainsi donc, de façon générale, nous nous référerons, dans les lignes qui suivent, à la documentation sous forme écrite.

0.3 Enfin, si l'on considère les documents produits par le travail terminologique, ils se répartissent en deux groupes :

a) les documents produits d̀ l'usage des terminologues;

b) les documents destinés aux usagers des terminologies.

Le schéma suivant illustre le procédé :

Cet article s'inspire largement d'un ouvrage en préparation par l'auteur : Introduction à la terminologie, à paraître en 1980 . 

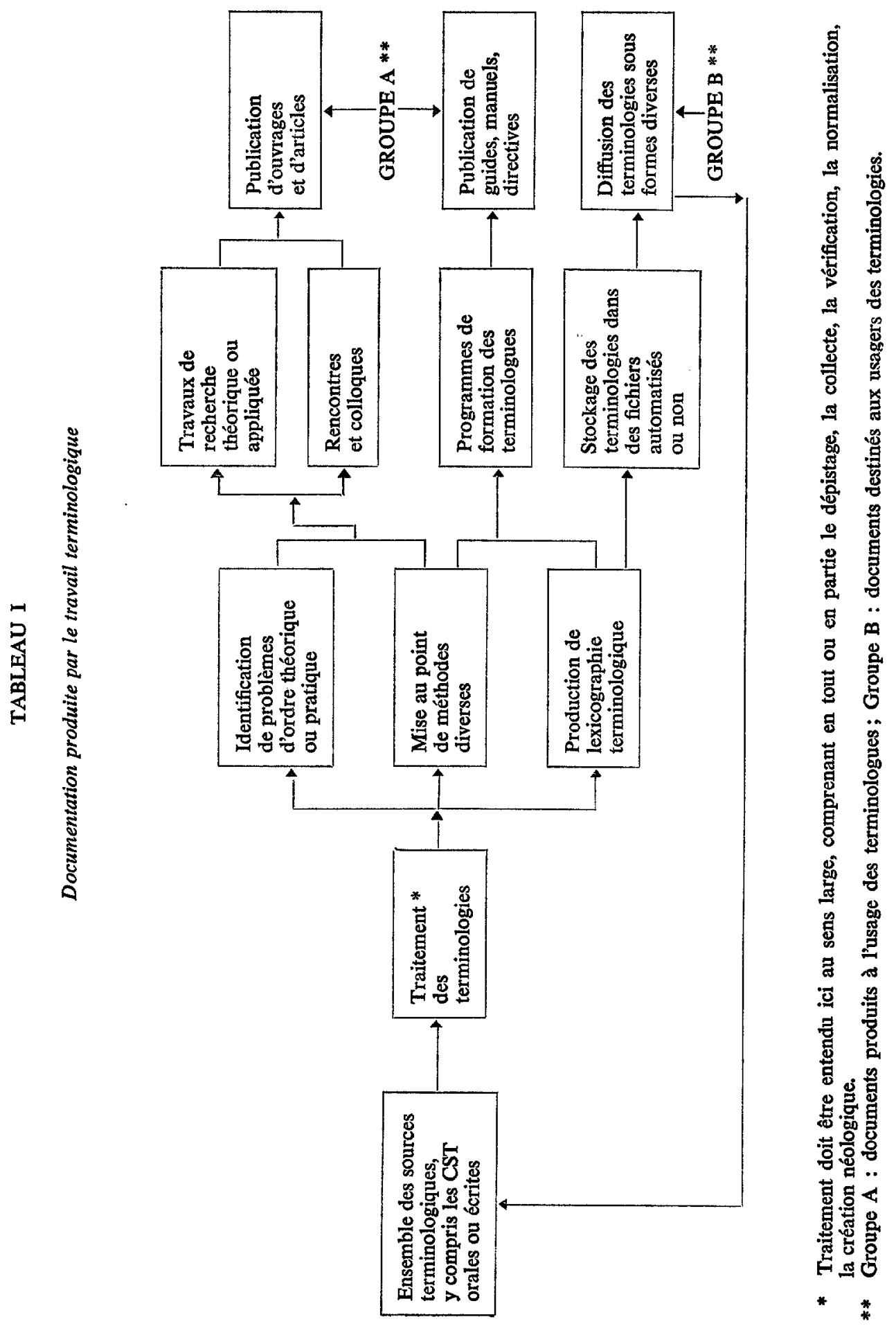
On peut constater que le premier groupe se subdivise à son tour en deux sous-groupes :

- celui qui comprend des ouvrages d'ordre théorique ou pratique, ainsi que des articles, exposés, communications dans des colloques ou congrès, etc., portant soit sur des questions proprement terminologiques (des questions relatives à des termes) soit sur des problèmes d'ordre méthodologique ou d'ordre linguistique au sens large rencontrés au cours des travaux terminologiques ;

- celui qui concerne plus particulièrement les méthodes de travail, y compris, par exemple, les normes et recommandations émanant du comité technique $\mathrm{n}^{0} 37$ de l'ISO.

Le second groupe de documents, outre qu'il se présente sous une très grande variété de formes, se caractérise par le fait qu'il sert à réalimenter le bloc «Ensemble des sources terminologiques».

\subsection{Typologie de la documentation}

L'ensemble documentaire terminologique (sources et outils ou sources et produits lexicographiques) peut se partager en cinq catégories qu'il convient de passer brièvement en revue.

1.1 Documentation relative à la recherche théorique et au développement de la discipline

Ces documents ont en général pour point de départ des problèmes rencontrés au cours des travaux terminologiques, comme le notaient Lotte et Wüster au début des années trente et comme l'ont observé de nombreux autres par la suite.

On y trouve des travaux de terminologues, de linguistes, de lexicographes et ils se caractérisent par le fait que s'ils trouvent leur origine dans les problèmes quotidiens que rencontre le terminologue, ils n'ont pas pour but, à court terme, de fournir au terminologue des recettes méthodologiques immédiatement applicables.

Leur objectif général est d'élaborer pour la terminologie une base théorique suffisamment large et solide sur laquelle pourront s'appuyer des travaux de recherche appliquée, constituant un ensemble cohérent, et orientés vers la recherche de solutions aux problèmes d'ordre pratique. C'est le propre de toute science $^{1}$, en effet, de se doter d'un cadre théorique, à partir du moment où son objet et ses méthodes commencent à se dessiner avec plus de précision. Le cas de la terminologie n'échappe pas à ce processus : née d'un besoin de précision et de clarté dans les langues de spécialité, elle s'est d'abord confondue avec la lexicologie et surtout la lexicographie pour s'en dégager par la suite en identifiant son objet propre et ses méthodes grâce à des moyens empiriques. On a fait de la terminologie bien avant de tenter de définir cette discipline.

1. Dubuc (1979), p. 15, refuse de considérer la terminologie comme une science; pour lui, elle n'est encore qu'un art, ce qui est tout à fait normal, compte tenu de la " notion de la terminologie » à laquelle il se réfère (p. 14). Cela démontre que l'appellation «terminologie » recouvre plusieurs notions, dont l'une correspond à ce que l'on est convenu d'appeler « art ou pratique » et l'autre à ce qui est à proprement parler une «science», avec un objet et des méthodes bien définis ou en voie de définition. 
Les quelques chercheurs qui se sont livrés à des réflexions sur la terminologie, encore que peu nombreux, ont jeté les bases d'une théorie de la terminologie dont on peut espérer des développements importants d'ici la fin $\mathrm{du}$ siècle.

Parmi les travaux à signaler dans cette catégorie, il faut mentionner :

- ceux de l'école germano-autrichienne

- ceux de l'école soviétique

- ceux de l'école tchécoslovaque

- ceux de l'école canado-québécoise

- ceux de chercheurs isolés (France, Grande-Bretagne).

Bon nombre de ces documents sont mentionnés dans les ouvrages cités en référence. Pour plus de détails, on pourra consulter la bibliographie établie par Rondeau, Felber et coll. (1979).

Nous nous limiterons, dans les lignes qui suivent, à une analyse des grandes tendances qui se dégagent de ces écoles, sur la question des fondements théoriques de la terminologie.

\subsubsection{Ecole germano-autrichienne}

Le principal représentant de cette école de pensée est $\mathrm{E}$. Wüster, ingénieur, industriel et professeur, qui vécut à Wieselburg et à Vienne. Parmi les autres noms, il faut également citer I. Dahlberg et G. Wersig ${ }^{2}$. Les publications de Wüster, qui débutent en 1931 avec sa thèse de doctorat, ont eu une influence considérable qui se maintient encore aujourd'hui. La pensée de Wüster est diffusée par l'un de ses premiers collaborateurs, H. Felber, directeur d'Infoterm ${ }^{3}$.

Parmi les grandes tendances de l'école germano-autrichienne, nous signalerons les suivantes, que nous énoncerons sous forme de postulats :

1. Le système notionnel est fondamental en terminologie; ce sont les notions que l'on classe d'abord, pour ensuite classer leurs dénominations.

2. Un système de notions est un système logique avec hiérarchisation structurale ; les notions se délimitent l'une par rapport à l'autre selon des rapports qui s'établissent sur deux axes : horizontal (compréhension) et vertical (extension). C'est sous cet aspect, notamment, que la terminologie participe de la logique et de l'ontologie (Wüster 1979). domaine.

En corollaire, un système de notions ne se définit que par rapport à un

3. L'univocité est une caractéristique fondamentale du terme, c'est-à-dire que le rapport qui s'établit entre notion et dénomination est toujours, en principe,

2. II faut également citer L. Hoffmann $(1976,1978)$ de l'Université Karl-Marx à Leipzig, dont les travaux, publiés en allemand, se rattachent surtout à l'école soviétique quant à la démarche.

3. Centre international d'information pour la terminologie. 
monoréférentiel ${ }^{4}$. D'où : a) rejet de la synonymie (sauf dans les cas d'exception) et b) nécessité de la normalisation terminologique.

Le principe de l'univocité conduit logiquement vers $l$ '« unification internationale des notions et des termes » (voir ISO/R860), la situation idéale étant représentée par les «mots internationaux $»$, unités terminologiques représentant la même notion et de forme à peu près identique dans plusieurs langues (ex. : chim. phys. : ion). Il conduit également vers des tentatives de création de langues artificielles.

4. La définition occupe en terminologie une place prépondérante : elle sert a délimiter la notion (ce n'est pas le terme qui fait l'objet de la définition). L'école germano-autrichienne doit principalement à $\mathrm{E}$. Wüster l'établissement de principes méthodologiques stricts, qui ont pour but de garantir une qualité uniforme des définitions terminologiques.

5. La documentation constitue un volet indispensable à tout travail terminologique. En corollaire, la science et les techniques de la classification (Dahlberg 1976) sont également indispensables.

6. La science terminologique (terminologielehre) s'applique à deux niveaux : celui de l'ensemble des terminologies et celui des terminologies particulières.

7. Dans le but de produire des terminologies servant effectivement à faciliter la communication, il faut normaliser les méthodes de la lexicographie terminologique.

\subsection{2 École soviétique}

Les travaux de l'école soviétique remontent, comme dans le cas de l'école germano-autrichienne, au début des années trente avec D.S. Lotte et S.A. Caplygin. Entre autres caractéristiques, on peut dire de cette école qu'elle comprend un nombre beaucoup plus important de représentants que dans le cas de la précédente. Parmi les chercheurs importants, il faut signaler Drezen, Vinokur, Kulebakin, Klimovitski, Kutina, Golovin, Kandelaki, Ahkmanova, Danilenko, etc. pour n'en mentionner que quelques-uns.

Parmi les constantes qui se dégagent de ces travaux, notons les suivantes :

1. La terminologie est une discipline appliquée en ce sens qu'elle cherche des solutions à des problèmes pratiques posés par la dénomination des notions dans les domaines scientifiques et techniques.

Ces problèmes se retrouvent en particulier : a) dans la normalisation terminologique en langue russe ainsi que dans les autres langues des républiques; b) dans la création néologique.

2. Comme l'objet de la terminologie est d'ordre langagier, les solutions devront faire appel à la linguistique. D'où :

4. Cette position extrême de l'école germano-autrichienne, inspirée des philosophes et des linguistes allemands, se conçoit facilement dans le cas des sciences exactes ou de nomenclatures systématiques; elle est irréaliste dès que l'on tombe dans d'autres domaines. Elle correspond au vieux rêve de la « langue parfaite » évoqué par Rey (1979, p. 42). 
a) une conception beaucoup plus linguistique de la terminologie ;

b) une influence moins profonde de la philosophie que dans l'école germanoautrichienne.

Cette conception se traduit d'ailleurs par une démarche différente au niveau des méthodes de travail. Alors que dans la démarche wüstérienne la classification des notions est préalable au classement et à la dénomination - il s'agit d'un exercice dans l'abstrait -, la démarche soviétique, au contraire ${ }^{5}$, construit un système notionnel à partir des éléments linguistiques (considérés par hypothèse comme termes) d'un domaine pour revenir par la suite à la vérification des notions.

3. Les problèmes terminologiques sont d'ordre socioculturel et sont dignes, par conséquent, de s'intégrer à la recherche universitaire. C'est ainsi que la plupart des chercheurs soviétiques en terminologie sont rattachés soit à une université, soit à l'Académie des sciences de l'URSS. Le premier colloque soviétique de terminologie, organisé par l'Académie des sciences, a eu lieu en 1959. Dès 1965, Mme Kandelaki donnait à l'université un cours de terminologie.

4. Les représentants de l'école soviétique ont dès les débuts manifesté un intérêt marqué à l'égard de problèmes fondamentaux, qui font d'ailleurs toujours l'objet de travaux de recherche dans les milieux terminologiques internationaux. Parmi ces problèmes, mentionnons :

- le découpage du terme

- la spécificité du terme (opposition terme/mot de la langue commune/ syntagme de discours)

- la notion en terminologie

- définition de la terminologie ; sa place parmi les autres disciplines

- distinction entre terminologie et nomenclature.

5. Dans le domaine de la normalisation terminologique, phénomène d'ordre sociolinguistique, l'école soviétique prône une attitude prudente et attentive qui n'est pas sans rappeler celle de la France et du Québec, qui ont établi une distinction importante entre la recommandation et la normalisation d'un terme ou d'un ensemble donné de termes.

\subsubsection{Ecole tchécoslovaque}

L'école tchécoslovaque a également été active dès le début des années trente, comme le note Kocourek (1965). Les travaux terminologiques sont étroitement liés à la défense de deux langues et de deux cultures, dichotomie qui se manifeste par l'établissement de l'Académie des sciences tchèque (Prague) et l'Académie des sciences slovaque (Bratislava). Parmi les représentants importants de l'école tchécoslovaque, il convient de citer : Havranek, Roudný, Drozd, Kocourek ${ }^{\circ}$.

\footnotetext{
5. Cette démarche se rapproche sur beaucoup de points de celle qui a cours à l'OLF, à

la DGTD et dans l'entreprise privée.

6. M. Kocourek enseigne depuis une dizaine d'années à l'Université Dalhousie, à Halifax.
} 
Les principaux thèmes abordés par les chercheurs tchécoslovaques sont :

1. La normalisation linguistique (y compris la normalisation terminologique) sur le plan national ainsi que la normalisation terminologique sur le plan international ?.

2. La considération des problèmes terminologiques dans une optique linguistique, non sans rapports d'ailleurs avec la tradition structuraliste de l'École de Prague.

3. La spécificité du terme et le type particulier de relation que le signe terminologique permet d'observer entre notion et dénomination.

\subsection{4 École canado-québécoise}

L'école canado-québécoise se distingue sous plusieurs aspects des trois précédentes; nous en énumérerons quelques-uns.

1.1.4.1 Il faut d'abord faire état de sa jeunesse. Ce n'est guère, en effet, que depuis le début des années soixante-dix que se développent les travaux terminologiques et que s'est amorcée, en conséquence, la réflexion sur ces travaux. Ce facteur, lié au fait que les travaux terminologiques ont été mis en route pour répondre à des problèmes pratiques urgents, explique sans doute que les principaux chercheurs de l'école canado-québécoise, plutôt que de se lancer dans l'élaboration de théories nouvelles, ont préféré dans une première étape, se livrer à une synthèse critique des travaux des quarante premières années et notamment de ceux de Wüster. Ils suivent en cela une courbe évolutive analogue à celle des deux principales écoles européennes, dont la pensée s'est élaborée au cours des années cinquante et soixante, à partir d'observations tirées des préoccupations d'ordre pragmatique et des travaux sur le terrain qui avaient marqué les deux décennies précédentes.

1.1.4.2 L'école canado-québécoise se caractérise de plus par son important dynamisme. Il suffit, pour s'en convaincre, de parcourir l'abondante liste des rencontres qui se sont échelonnées depuis 1972 , de prendre connaissance des nombreuses réalisations à Ottawa, Québec et Montréal notamment, dans de multiples aspects de la terminologie, et de consulter la liste des cours de terminologie qui se donnent dans différentes universités.

1.1.4.3 L'école canado-québécoise n'est pas renfermée sur elle-même. Au contraire, elle alimente sa réflexion en faisant appel à des contributions internationales, comme le font voir les colloques internationaux qui se sont tenus au Québec sous l'égide de la Régie, puis de l'Office de la langue française.

1.1.4.4 A l'intérieur de l'objectif général de l'amélioration de la communication dans les domaines de la science et de la technologie, l'école canadoquébécoise poursuit un double objectif, dichotomie marquée d'ailleurs dans les faits par une concentration sur deux pôles des travaux terminologiques. Pour la

7. Après une période de participation assez intense aux activités de normalisation terminologique internationale de l'après-guerre, la Tchécoslovaquie, depuis le début des années soixante, semble marquer le pas. 
Direction générale de la terminologie et de la documentation d'Ottawa, de même qu'en général pour le groupe de Montréal, la vocation principale des travaux terminologiques est d'ordre traductionnel; pour la Direction de la terminologie de l'Office de la langue française du Québec ainsi que pour le groupe qui s'y rattache (UQTR, UQAM, etc.) la terminologie est essentiellement un outil d'ordre sociolinguistique qui doit concourir à l'implantation du français comme langue de travail ( $=$ des activités scientifiques, techniques ou professionnelles).

1.1.4.5 Enfin, les priorités de l'école canado-québécoise sont orientées, dans l'ordre, vers le développement des méthodes de travail, vers la formation des terminologues (et terminographes) et vers la formation de principes directeurs destinés à assurer aux travaux terminologiques une saine cohésion.

\subsubsection{Chercheurs isolés}

Il convient d'abord de signaler, en France, Louis Guilbert, que l'on peut sans aucun doute qualifier de maître à penser de la terminologie française. C'est dans une optique à la fois linguistique et parfois socialisante que Guilbert envisage la terminologie. Disparu trop tôt pour avoir eu le temps d'élaborer sa conception d'une théorie de la terminologie, il n'en a pas moins laissé des fragments importants d'un système cohérent.

Il faut citer également, en France, les travaux d'Alain Rey, qui présente de la terminologie une conception inspirée à la fois de la philosophie et de la lexicographie.

En Grande-Bretagne, J.C. Sager, bien au courant des travaux de l'école germano-autrichienne, envisage cependant la terminologie sous un angle plus linguistique que Wüster.

1.2 Documentation relative aux postulats fondamentaux sur lesquels s'appuient les travaux terminologiques

Cette catégorie de documents se caractérise par le fait qu'elle regroupe des textes dont l'objectif est d'ordre pratique et immédiat, sans toutefois entrer dans le détail des méthodes de travail. Ces documents ne contiennent pas non plus de discussions relatives à des problèmes théoriques.

Ils traduisent en général un consensus, fruit de discussions plus ou moins longues entre praticiens, et ont pour objectif l'harmonisation des méthodes de travail, compte tenu de la diversité des buts à atteindre et, par conséquent, de la souplesse à conserver dans l'application des postulats fondamentaux. national.

On peut considérer ces données documentaires sur les plans international et

1.2.1 Sur le plan international, il faut mentionner les normes et recommandations émanant du Comité technique $n^{\circ} 37$ de l'ISO $^{8}$ (Organisation internationale de

8. \&... l'ISO a constitué un Comité Technique dont la mission est de dégager et de formuler des principes généraux en matière de terminologie et de lexicographie terminologique : le Comité Technique ISO/TC 37, Terminologies (Principes et coordination). 
normalisation). Ce «Comité technique » a pour mandat général d'élaborer des principes et d'assurer la coordination en ce qui concerne les travaux terminologiques des multiples ${ }^{2}$ unités de travail de l'ISO, qui comprend 86 pays membres.

Les documents publiés à ce jour sont :

- ISO R 704 Principes de dénomination

- ISO R 860 Unification internationale des notions et des termes

- ISO R 1087 Vocabulaire de la terminologie

Ces documents sont en révision; la coordination des travaux de révision a été confiée au groupe ${ }^{10}$ de travail 1 de l'ISO/CT 37 (secrétariat : Moscou).

1.2.2 Sur le plan national ${ }^{11}$, on peut citer comme exemples les Actes du Colloque international de terminologie tenu à Baie St-Paul en 1972, au cours duquel a été élaborée la liste des éléments (essentiels et facultatifs) devant normalement faire partie d'un dossier terminologique. C'est à partir de cette liste que, par exemple, les deux banques de terminologies du Canada et du Québec ont structuré leurs fichiers.

Il faut également faire mention des principes directeurs adoptés lors du Colloque canadien sur les fondements d'une méthodologie générale de la recherche et de la normalisation en terminologie et en documentation tenu à Ottawa en 1976 (Rondeau, réd. 1976, 1979a), des Actes du colloque international sur la normalisation linguistique (OLF 1974), des études sur la néologie que l'on retrouve dans Hamelin $(1975,1977)$ ainsi que dans le chapitre d'introduction de Néologie en marche (Boulanger, réd. 1978).

1.3 Documentation relative aux méthodes de traitement terminologique

Encore ici, il y a lieu de distinguer l'un de l'autre les deux plans international et national ; on retrouve, dans les deux cas, deux sous-catégories :

- celle des guides de travail ;

- celle des cours et programmes de formation.

Les publications élaborées par ce Comité Technique concernent 4 catégories de questions, à savoir :

1. Vocabulaire de la terminologie ;
2. Processus d'élaboration des vocabulaires normalisés nationaux et internationaux;

3. Normalisation nationale et internationale des notions, des termes et de leurs définitions : principes de formation et critères de valeur ;

4. Présentation des vocabulaires monolingues et multilingues, y compris les symboles lexicographiques.

La publication ressortissant à la catégorie 2 constitue une sorte de guide pour l'organisation du travail, tandis que celles des autres catégories concernent les détails techniques.

Extrait de l'avant-propos de Norme internationale ISO 1951, ISO, Genève, 1973.

9. On compte à l'heure actuelle plus de 165 comités techniques de 1'ISO, dont la plupart ont mis sur pied un sous-comité ou des groupes de travail consacrés à la terminologie du domaine propre au comité technique. Signalons également le groupe de travail 1 de la Commission électrotechnique internationale (CEI), dont le mandat est strictement d'ordre terminologique.

10. Le groupe de travail 1 deviendra bientôt le sous-comité 1 de l'ISO/CT 37.

11. Nous nous en tiendrons, dans le cadre de cet article, au plan « national défini comme le territoire géographique du Canada actuel, sans entrer dans le détail des travaux des nations étrangères. 
1.3.1 Sur le plan international, on peut citer comme exemples les documents suivants, dans la catégorie des guides de travail :

- ISO/R 919 Guide pour l'élaboration des vocabulaires systématiques (exemple de méthode)

- ISO/R 1149 Présentation des vocabulaires systématiques multilingues

- ISO/DP 4466 Présentation des vocabulaires systématiques monolingues

- ISO/1951 Symboles lexicographiques

- ISO/R 639 Indicatifs de langue, de pays et d'autorité

Dans la sous-catégorie des cours et programmes de formation, on trouve; outre les stages de formation envisagés par le CT 37 de l'ISO à l'intention des groupes de travail en terminologie des différents Comités techniques de l'ISO, des stages régionaux comme par exemple celui de Nordterm (Picht 1979) en Scandinavie. On peut également citer les programmes prévus par l'ONU, qui vient, en 1978, de former à New York un groupe de travail avec mandat d'étudier la possibilité de création d'une banque de termes.

1.3.2 Sur le plan national, dans la sous-catégorie des guides de travail, on trouve de nombreux exemples, parmi lesquels il faut citer :

- Guide de travail en terminologie (OLF 1973)

- Méthodologie de la recherche terminologique (Auger, Rousseau 1978)

- Guide de rédaction de la fiche Termium II (DGTD 1978)

- Guide de recherche terminologique (CN/AC 1978) ${ }^{12}$

Dans la sous-catégorie des cours et programmes de formation, les exemples les plus anciens à citer sont le cours de Kandelaki (voir par. 1.1.2) et celui de Wüster, réédité récemment en allemand (Wüster 1979) et bientôt disponible également en français et en anglais. Mais il y a de nombreux autres cours en marche depuis quelques années, dont on trouvera une liste dans les Actes du colloque international sur l'enseignement de la terminologie (Rondeau, réd. 1979).

Une brève énumération de ces cours, bien qu'incomplète, suffira à montrer l'ampleur des activités dans ce domaine : programmes de deuxième cycle et cours d'introduction dans une demi-douzaine d'universités canadiennes, programmes de formation structurés dans les organismes gouvernementaux canadiens, cours d'introduction dans plusieurs universités européennes, notamment à Vienne, Copenhague, Saarbrücke; programmes de formation sur le tas dans des organismes internationaux comme la C.E.E. à Bruxelles et à Luxembourg, ainsi que dans des multinationales comme Siemens, dans des entreprises comme Bell Canada, etc.

Ces cours et programmes de formation varient considérablement dans leur formule et dans leur contenu, selon les besoins du milieu dans lequel ils se donnent et selon les disponibilités du personnel enseignant. Il faut noter toutefois

12. Je suis reconnaissant à $\mathbf{N}$. Bélanger de m'avoir transmis ce document qui, bien que sous une forme encore provisoire, démontre l'intérêt des entreprises à l'égard de la formation des terminologues. 
des efforts sur le plan international vers la définition d'un contenu minimal commun. Ces efforts ont été amorcés à Vienne en 1977 dans une recommandation émanant d'un atelier sur la terminologie organisé dans le cadre du Premier colloque international sur la terminologie et les Lsp (AILA); cette recommandation a donné lieu à la tenue à Québec du Colloque international sur l'enseignement de la terminologie en 1978 ; l'une des recommandations de ce colloque porte sur la création d'un comité international de rédaction d'un ouvrage général destiné à l'enseignement de la terminologie. Enfin, le thème de l'enseignement de la terminologie a été repris à Moscou en novembre 1979, lors du Symposium international sur la terminologie organisé conjointement par l'Académie des sciences de l'URSS, le VNIIKI, Infoterm et l'AILA.

\subsection{Documentation à contenu terminologique}

Cette catégorie comprend les documents dans lesquels se retrouvent des terminologies, soit en contexte, soit hors contexte et, dans ce dernier cas, classées selon des modes divers. Certains de ces documents ne contiennent que des termes; d'autres contiennent à la fois des termes et des mots de la langue commune.

Ces documents se répartissent en huit sous-catégories :

1.4.1 Normes internationales et nationales portant sur des produits et procédés (ISO, CEI, ACNOR, AFNOR, BSI, VNIIKI, ON, DIN, etc.). L'ensemble des termes contenus dans ces normes peut donner lieu, comme c'est le cas pour l'AFNOR, à la mise sur pied d'une banque de termes.

1.4.2 Manuels, catalogues, modes d'emploi, directives, guides de l'usager, etc.

1.4.3 Livres et revues spécialisées, actes de rencontres scientifiques, rapports de recherche, etc.

1.4.4 Vocabulaires, thésaurus, glossaires, lexiques, etc.

1.4.5 Dictionnaires généraux et spécialisés, de langue ou encyclopédiques, encyclopédies, etc.

1.4.6 Banques de termes, fichiers automatisés ou non.

1.4.7 Consultation de spécialistes d'un domaine.

1.4.8 Bibliographies ou listes portant sur ces sous-catégories.

1.5 Documents d'information et de coordination produits par divers organismes et réseaux

On trouve par exemple, parmi ces organismes, Infoterm, TermNet, Nordterm, Hispanoterm, Afterm, AILA-Comterm, etc.

Enfin, nous présentons en annexe un « arbre de la documentation terminologique $\gg$ (Tableau II) qui fournit une vue d'ensemble de tous les types de documents mis à la disposition des usagers, médiats et immédiats, des terminologies.

2.0 Problèmes d'évaluation des sources documentaires (paragraphe 1.4) dans les travaux terminologiques 
Dans la catégorie des documents terminologiques, l'un des problèmes les plus difficiles à résoudre, non seulement pour le terminologue chevronné, mais à plus forte raison pour le terminologue débutant, est celui de l'évaluation des sources. La qualité des terminologies réunies en vocabulaires spécialisés est, en effet, fonction de la qualité des documents desquels elles sont extraites. Or, la valeur de ces documents varie considérablement en fonction de facteurs comme la qualité de l'auteur, l'âge du document, le type de lecteur à qui il s'adresse, etc. Il n'existe aucun ensemble structuré de critères permettant d'évaluer la qualité linguistique de la documentation scientifique et technique, de sorte que traducteurs, terminologues, rédacteurs techniques et professeurs de Lsp doivent établir individuellement et de façon empirique les éléments sur lesquels ils fondent leurs jugements.

\subsection{Qualité des termes}

De plus, la qualité d'un terme doit être évaluée à la fois sur le plan scientifico-technique et sur le plan linguistique.

Sur le plan scientifico-technique, la question qui se pose est de savoir si telle dénomination correspond bien, dans l'esprit des spécialistes du domaine, à telle notion; s'il se trouve plus d'une dénomination pour la même notion, s'agit-il de véritables synonymes terminologiques? C'est le critère de la valeur notionnelle propre du terme.

Sur le plan linguistique, il y a deux aspects à considérer :

a) celui de la qualité linguistique interne, c'est-à-dire de la conformité de l'appellation (forme extérieure du terme) aux règles morphologiques, syntaxiques, graphiques, stylistiques même, de la langue dans laquelle le terme est exprimé;

b) celui de la valeur notionnelle comparée, que l'on rencontre en terminologie bilingue ou multilingue. Il s'agit de vérifier si tel terme d'une langue donnée est ou non l'équivalent ( $=$ renferme la même notion) que tel terme dans une autre langue. C'est sous cet aspect que les CST obtenues par traduction ainsi que les ouvrages lexicographiques bilingues ou multilingues doivent être utilisés — quand il le faut -, avec une grande prudence.

\subsection{Qualité des sources}

Lors du colloque d'Ottawa (voir plus haut, 1.2.2), un groupe de travail a abordé la question des critères d'évaluation des sources documentaires (Rondeau, réd. 1976,1979 ). Il s'agit, semble-t-il, de la première - et probablement de l'unique - tentative d'aborder systématiquement la question ; aussi n'est-il pas inutile de résumer l'ensemble des critères dégagés par les participants.

Il y a lieu, d'abord, d'établir une distinction entre :

a) les ouvrages lexicographiques et les vocabulaires terminologiques; et

b) les travaux non lexicographiques :

ba) d'ordire pédagogique

bb) à valeur informationnelle (CST). 


\subsubsection{Ouvrages lexicographiques}

Dans le premier cas, les critères les plus importants à considérer sont :

1. la méthode de production de l'ouvrage. La méthode de collecte et de traitement des mots (ouvrages lexicographiques) ou des termes (vocabulaires spécialisés) est-elle clairement exposée ? Le cas échéant, comment se conforme-t-elle à une méthodologie généralement considérée comme sûre par les organismes internationaux et nationaux?

2. la présence d'un système de renvois entre les unités linguistiques appartenant au même champ notionnel ;

3. la présence d'un index alphabétique, notamment dans le cas des ouvrages de lexicographie terminologique systématique;

4. le mode de présentation des données; permet-il une consultation facile de l'ouvrage?

5. la présence d'illustrations et, le cas échéant, leur qualité ;

6. la présence de définitions ou, à défaut, de contextes variés permettant une délimitation précise des notions;

7. la présence d'indications de sources;

8. une délimitation précise du domaine pour chaque terme.

\subsubsection{Ouvrages non lexicographiques}

Dans le second cas et pour les deux sous-catégories, il faut tenir compte des aspects suivants :

1. la langue dans laquelle est rédigé le texte; s'agit-il d'une traduction ou d'un texte en langue d'origine? s'il s'agit d'un texte en langue d'origine, cette langue est-elle ou non une langue étrangère pour l'auteur ?

2. l'auteur (personne physique ou morale) est-il une autorité reconnue dans son domaine?

3. la date de la publication et des mises à jour; la dernière mise à jour est-elle récente ? s'agit-il d'une véritable mise à jour ou d'une simple réimpression?

4. le lecteur à qui s'adresse le document est-il considéré comme un spécialiste du domaine, ou est-ce qu'il s'agit d'un ouvrage de vulgarisation ?

5. le niveau de langue est-il homogène, par rapport au critère précédent ?

6. l'ouvrage s'adresse-t-il à un public géographiquement limité ou bien est-il neutre du point de vue des régionalismes?

7. l'éditeur ou, dans le cas des périodiques, le comité de rédaction offre-t-il des garanties suffisantes de qualité ?

Voilà un ensemble de critères que l'on peut appliquer à priori et qui permettront, dans beaucoup de cas, de porter un jugement de valeur assez juste sur un document à teneur terminologique.

Ces critères ne devraient, cependant, être considérés que comme le point de départ de l'établissement d'une grille d'évaluation qui permettrait d'aller beaucoup plus loin dans l'appréciation des sources documentaires. 


\section{TABLEAU II}

Arbre de la documentation terminologique

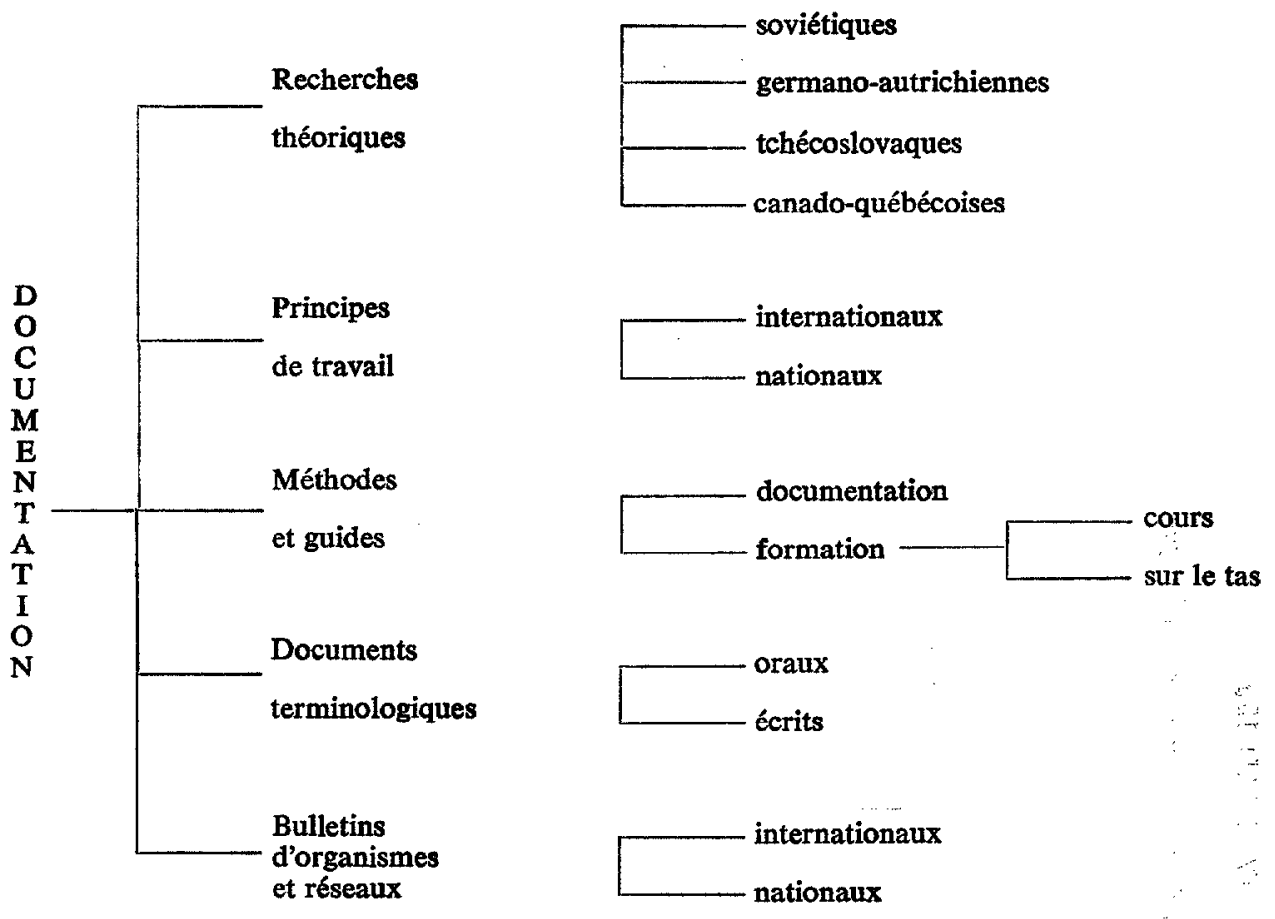



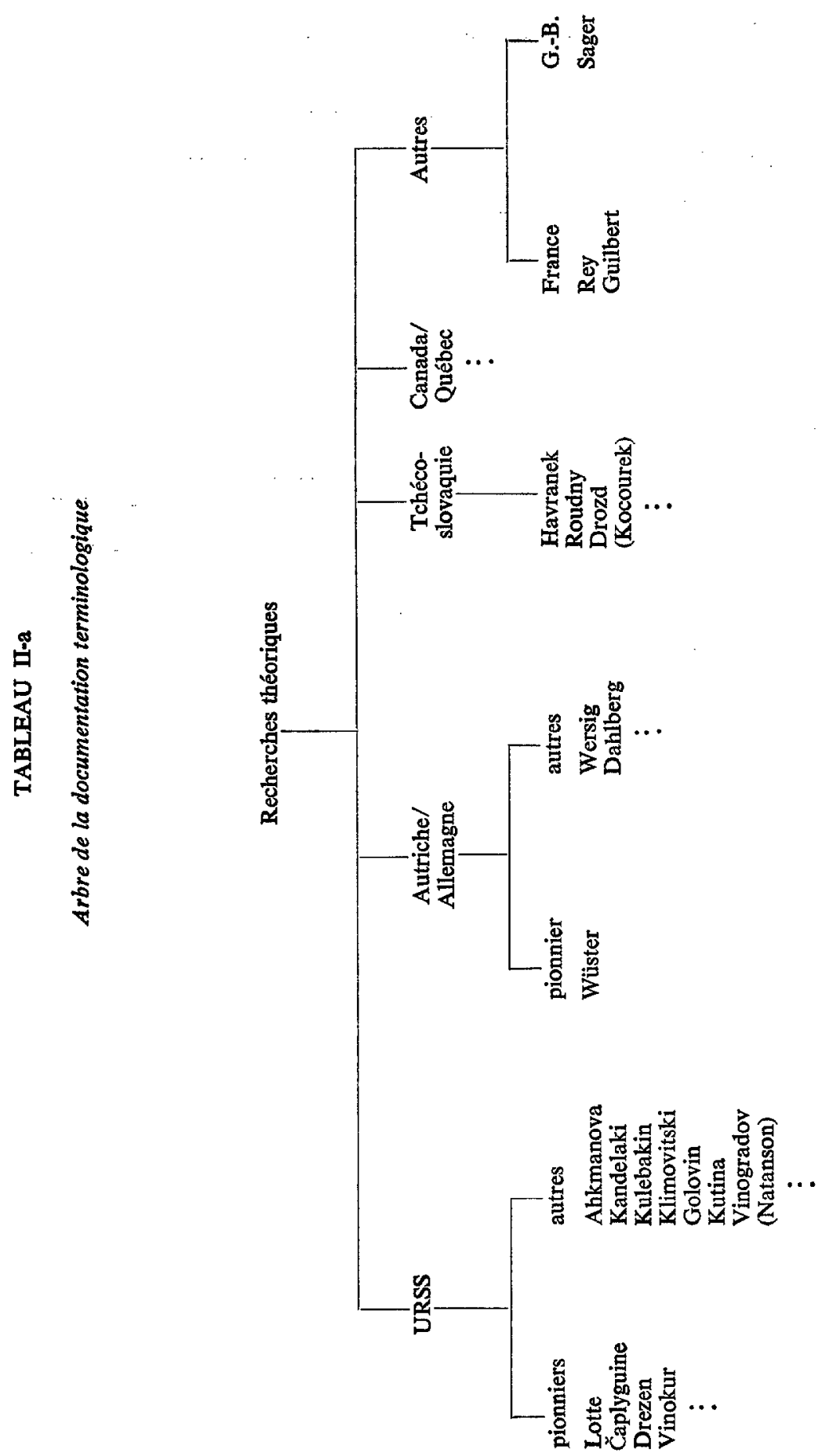

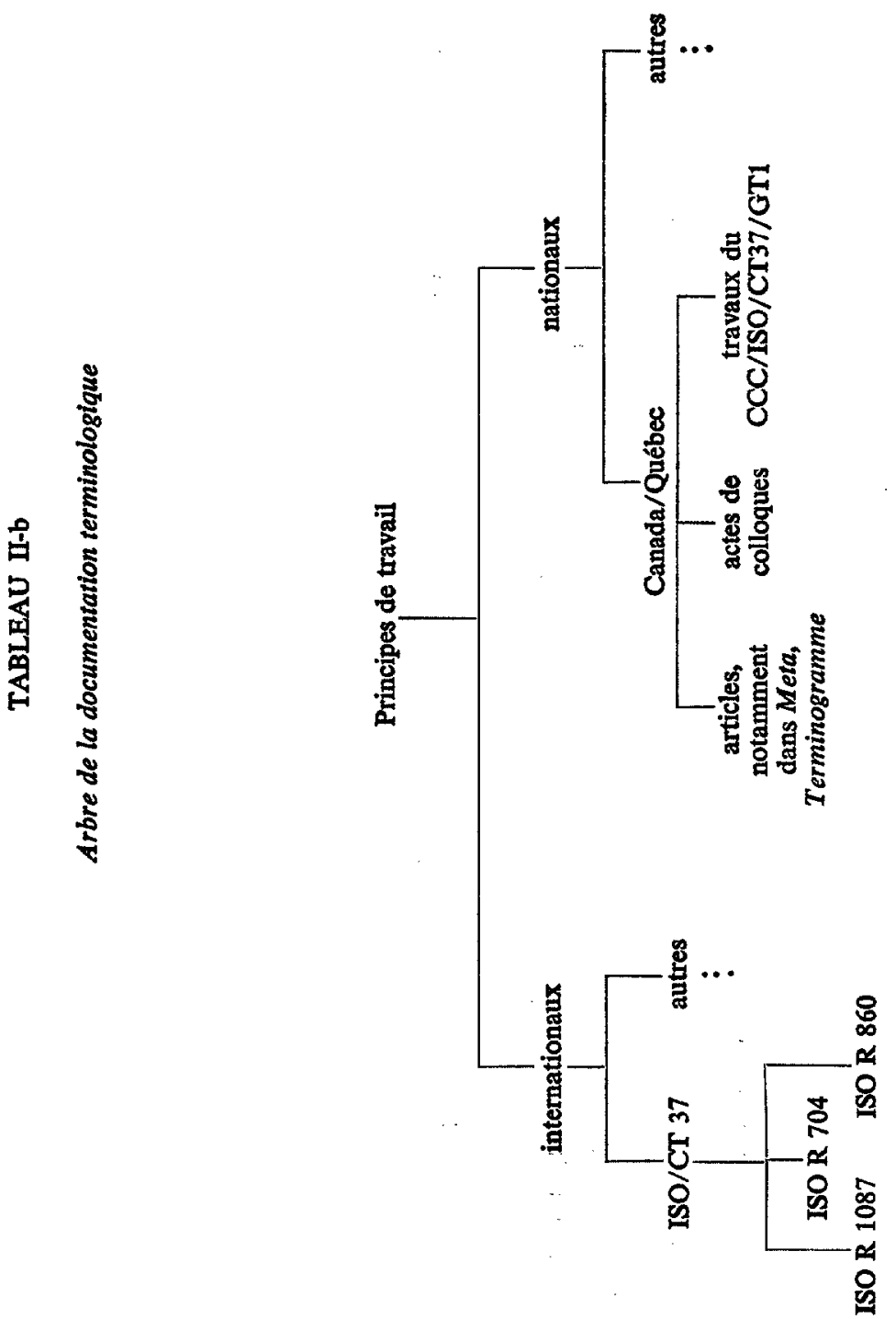


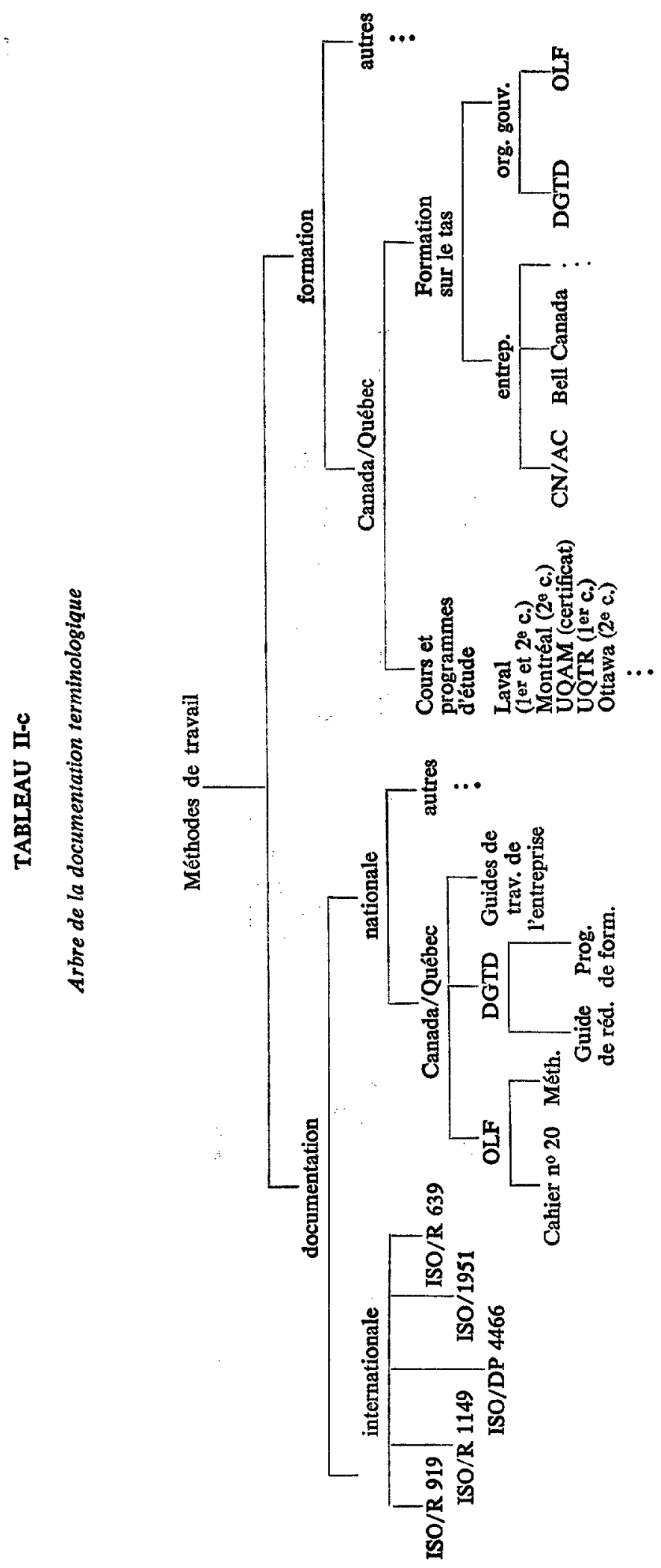




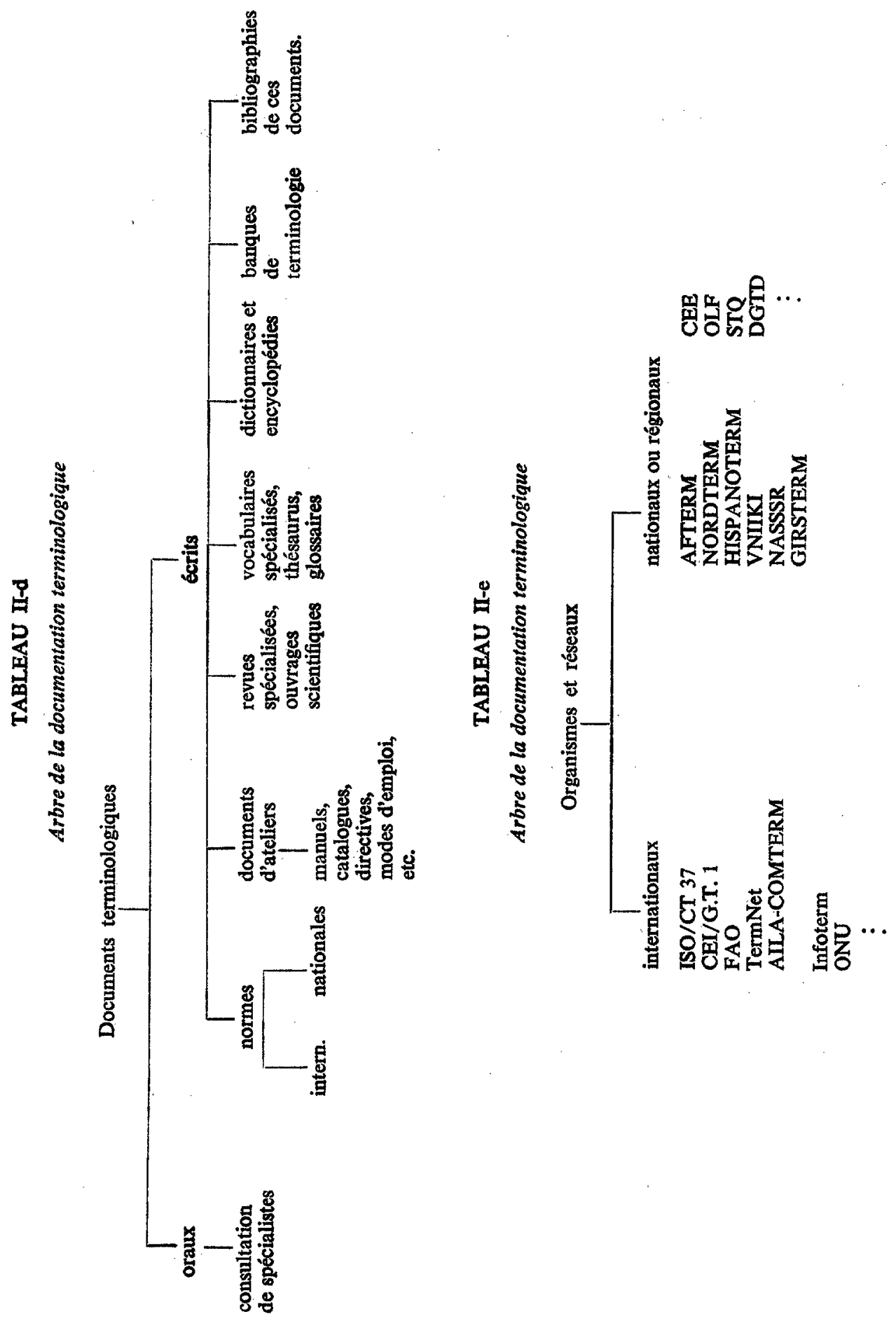




\section{OUVRAGES CITÉS EN RÉFÉRENCE}

AUGER, P., ROUSSEAU, L.-J. et COLL. (1978), Méthodologie de la recherche terminologique, Office de la langue française, Éditeur officiel du Québec.

BOULANGER, J.C., réd. (1978), Néologie en marche, série b : langues de spécialité, no 4, Office de la langue française, Éditeur officiel du Québec.

$\mathrm{CN} / \mathrm{AC}$ (1978), Guide de recherche terminologique (document provisoire), Service de linguistique CN/AC, section de terminologie, Montréal.

DAHLBERG, I. (1976), Über Gegenstande, Begriffe, Definitionen und Benennungen (Les objets, les notions, les définitions et les termes) in Muttersprache, Sonderdruck 2, Gesellschaft für Deutsche Sprache, Wiesbaden.

DGTD, Direction générale de la terminologie et de la documentation (1978), Guide de rédaction de la fiche Termium II, Ottawa.

DUBUC, R. (1979), Manuel pratique de terminologie, Linguatech, Montréal.

HAMELIN, L.-E. (1975), «De la néologie en géographie », Cahiers de Géographie de Québec, no 48, P.U.L., Québec.

HAMELIN, L.-E. (1977), « Le Nord et son langage », dans Néologie en marche, série b : langues de spécialités, no 5 , fascicule 1 , Office de la langue française, Éditeur officiel du Québec.

HOFFMANN, L. (1976), Kommunikationsmittel Fachsprache, Eine Einführung, Sammlung Akademie-Verlag, 44, Akademie-Verlag, Berlin (Languages for Special Purposes as a Means of Communication : An Introduction).

HOFFMANN, L. (1978), Sprache in Wissenschaft und Technik, Ein Sammelband, VEB Verlag Enzyklopädie Leipzig, Leipzig.

ISO/R860 (1968), Unification internationale des notions et des termes, ISO, Genève.

KOCOUREK, R. (1965), "Termin a jeho definice (Le terme et sa définition), dans Čekoslovensky terminologicky časopis, vol. IV, no 1 .

OLF : Office de la langue française (1973), Guide de travail en terminologie, Cahiers de l'Office de la langue française, no 20 , Gouvernement du Québec.

OLF : Office de la langue française (1974), La normalisation linguistique, Actes du colloque international de terminologie, réd. M. Héroux, Office de la langue française, Lac Delage (Québec, 16-19 octobre 1973), Éditeur officiel du Québec.

PITCH, H. (1979), «Nordic Seminar on Terminology 》, dans COMTERM, Bulletin de la Commission de terminologie de l'AILA, no 2, AILA-Comterm, Québec.

REY, A. (1979), La terminologie : noms et notions. Coll. Que sais-je ?, no 1780, P.U.F., Paris.

RONDEAU, G., réd. (1976), Actes du colloque canadien sur les fondements d'une méthodologie générale de la recherche et de la normalisation en terminologie et en documentation, février 1976, DGTD, Ottawa (nouvelle édition GIRSTERM/DGTD, 1979).

RONDEAU, G., réd. (1979), Actes du colloque international sur l'enseignement de la terminologie, Office de la langue française, Québec, août 1978, Éditeur officiel du Québec.

RONDEAU, G., réd. (1979a), Actes du colloque canadien sur les fondements d'une méthodologie générale de la recherche et de la normalisation en terminologie et en documentation, GIRSTERM/DGTD, Québec et Ottawa (réédition des Actes parus en 1976).

RONDEAU, FELBER et COLL. (1979), Bibliographie internationale de la terminologie, GIRSTERM, Université Laval, Québec.

WÜSTER, E. (1979), Einfïhrung in die Allgemeine Terminologielehre und Terminologische Lexicographie, Schriftenreihe der Technischen Universität Wien, in Kommission bei Springer-Verlag, Wien/New York (voir 1979a, et 1979b).

WÜSTER, E. (1979a), Introduction à la théorie générale de la terminologie et à la lexicographie terminologique (traduit de Einführung in die Allgemeine Terminologielehre und Terminologische Lexicographie), GIRSTERM, Université Laval, Québec (traduction française : Bureau des traductions, Secrétariat d'État, Canada).

WÜSTER, E. (1979b), Introduction to the General Theory of Terminology and Terminological Lexicography, (translated from Einführung in die Allgemeine Terminologielehre und Terminologische Lexicographie), GIRSTERM, Université Laval, Québec (English translation : Translation Bureau, Secretary of State, Canada). 